\title{
TEM Specimen Preparation Using a Low Energy Ion Beam for Nuclear Metallic Materials
}

\author{
Joven J H Lim ${ }^{1}$, Eric Prestat ${ }^{2,3}$, Quentin Ramasse ${ }^{3,4,5}$, and M Grace Burke ${ }^{2}$ \\ 1. UK Atomic Energy Authority, Culham Science Centre, Abingdon, OX14 3DB, UK \\ 2. Materials Performance Centre, University of Manchester, Oxford Road, Manchester, M13 9PL, UK \\ 3. SuperSTEM Laboratory, SciTech Daresbury Campus, Daresbury, WA4 4AD, UK \\ 4. School of Physics, University of Leeds, Leeds LS2 9JT, UK \\ 5. School of Chemical and Process Engineering, University of Leeds, Leeds LS2 9JT, UK
}

Traditional TEM specimen preparation techniques such as electropolishing for nuclear metallic materials irradiated by either accelerated ions or neutrons are not optimum for producing TEM specimens for advanced (S)TEM analysis due to three main reasons: (1) the difficulty in preparing specimens from a specific site of interest, (2) lack of control in obtaining an ideal thickness at the electron-transparent region of interest, and (3) the time-consuming process to generate new electropolishing "recipes" for new nuclear alloys that are being developed for next-generation nuclear reactor systems. In order to overcome these issues, the Focused Ion Beam (FIB) technique is used to prepare TEM specimens from nuclear metallic materials. Such a technique is ideal for preparing TEM specimens from a specific region of interest in a radioactive nuclear material to study the microstructural evolution that resulted in materials properties degradation after exposure to high energy radiation. The radioactivity of the FIB lamellae will be significantly reduced and will ease the handling of such TEM specimens. Also, unlike the electropolishing technique, hundreds of FIB lamellae can be extracted from the same volume that is required to prepare one TEM specimen using electropolishing technique. This can significantly reduce the total experimental cost by reducing the volume of nuclear materials to be irradiated at either an ion beam facility or test nuclear reactor.

However, one fundamental drawback of using FIB to prepare TEM specimens from an irradiated nuclear metallic material is that FIB induces cascade damage into the sub-surfaces of TEM specimens during the milling/polishing process. Such damage is identical to the damage induced by high energy ion/neutron irradiation. Hence, the FIB-induced cascade damage will affect both quantitative and qualitative results obtained, especially for dislocation loops analyses from the irradiated nuclear metallic system and will indirectly underestimate the radiation resistivity of nuclear metallic material. In this study, a TEM specimen preparation technique using both FIB and low energy ion beams has been developed to reduce the FIB-induced damage in nuclear metallic materials. The metallic systems involved in this study are mainly BCC and FCC alloys. A $200 \mathrm{kV}$ FEI Tecnai T20 analytical TEM with a $\mathrm{LaB}_{6}$ cathode was used to characterize the defects induced by low energy ion beams in the $\mathrm{BCC}$ and FCC alloys. The significant improvement in quality of the TEM specimens prepared by using this newly developed technique, as shown in Figure 1, will be discussed in detailed.

\section{References:}

[1] The sample preparation equipment used UKAEA's Materials Research Facility, which has been funded by and is part of the UK's National Nuclear User Facility and Henry Royce Institute for Advanced Materials [Grant No. EP/P021727/1] 
[2] This work has been supported by the RCUK Energy Programme [Grant No. EP/P012450/1] and EPSRC Grant No. EP/N017854/1, and has been carried out within the framework of the EUROfusion Consortium and has received funding from the Euratom research and training programme 2014-2019 under Grant Agreements No. 633053. The views and opinions expressed herein do not necessarily reflect those of the European Commission. To obtain further information on the data and models underlying this abstract please contact PublicationsManager@CCFE.ac.uk

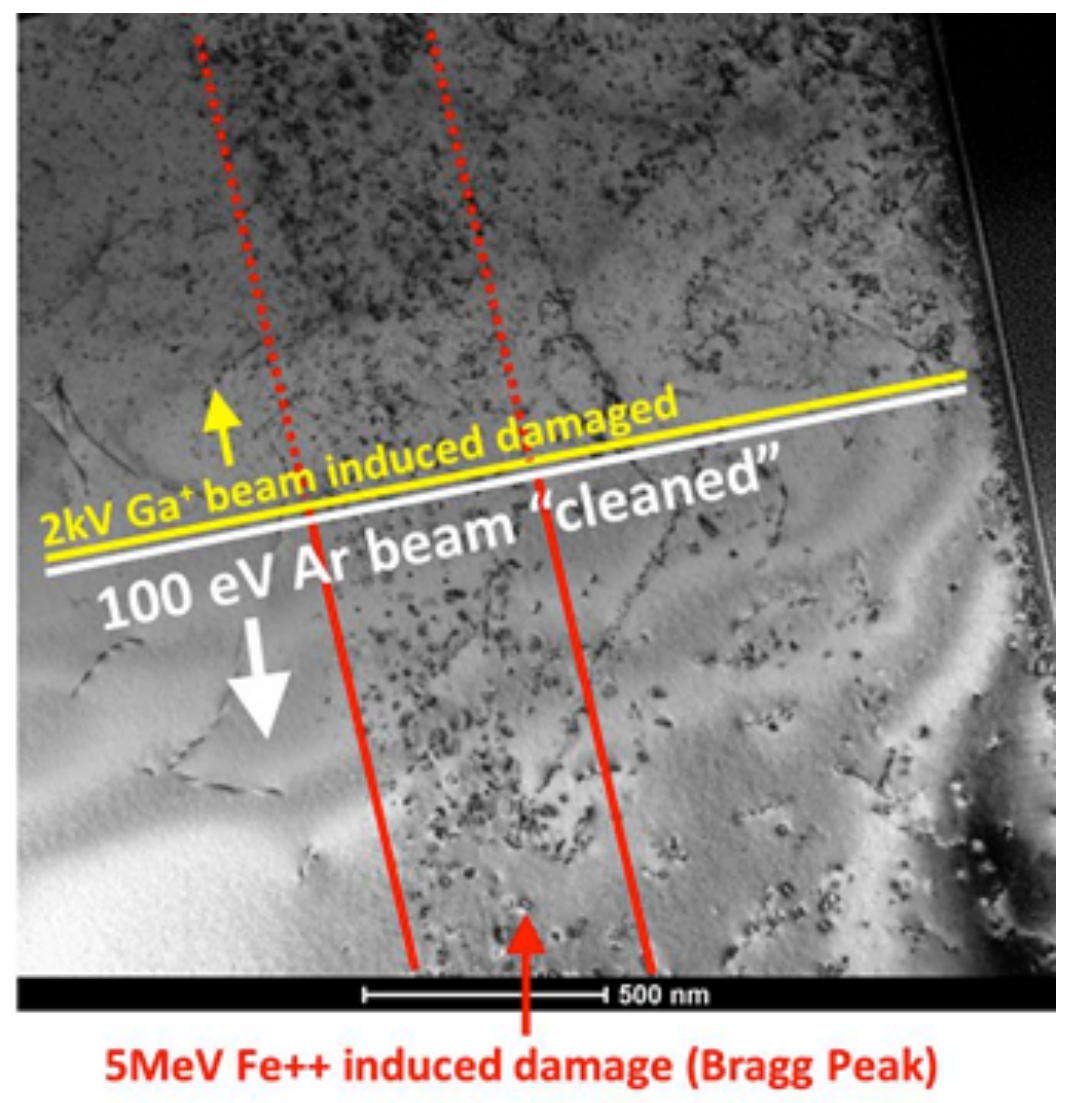

Figure 1 (Left). CTEM Kinematic Bright Field (KBF) micrograph imaged from an ion irradiated FCC $\mathrm{FeNiCr}$ alloy, i.e. Alloy $800 \mathrm{H}$, up to $1 \mathrm{dpa}$ showing a significant improvement in specimen quality after post-Focused Ion Beam (FIB) ion polishing process, i.e. $100 \mathrm{~V}$ Ar ion-beam milling. KBF micrograph imaged both the high energy ion irradiated region and non-irradiated region. The Bragg peak damage induced by the high energy ion beam is shown by the red arrow. The upper region of the KBF micrograph, the region above the yellow solid line, corresponds to a region that received no post-FIB ion polish. The lower region of the KBF micrograph, the region below the white solid line, showed the region that had undergone post-FIB ion polishing. This KBF micrograph reveals the significant improvement of the quality of the TEM specimen after the post-FIB ion polishing. 